\title{
Experiencia en hemorroidectomías aplicado en la Clínica Fiori.
}

\author{
ALIAGA CHAVEZ Noél*; AMARO GOMEZ SANCHEZ Miguel; SANCHEZ ZAPATA José Antonio***
}

\section{SUMMARY}

Objective: To evaluate the utility of the ambulatory surgery with local anesthesia in patient with hemorrhoidal pathology. Material and methods: A prospective, transverse study was performed between April 1996 and March 1999, in the division of Surgery of the Clinica Fiori. We intervened 260 patients with hemorrhoidal pathology, of them 229 patients were haemorrhoidectomys with local anesthesia, those that included in the study. The used surgical technique was that of Millígan and Morgan with an operative time of 20 minutes; and control to the 3 days. Results: $69.4 \%$ of hemorrhoidal pathology was observed among the 3160 years of age. Only $1.7 \%$ (4 patients) they presentes post-operative complications, 2 patients with having bled light and 2 with residual hemorrhoids, none was being of graveness; and $98.3 \%$ (225 patients) they didn't present any type of complications. There were not anesthetic accidents. Conclusion: The haemorrhoidectomy with anesthesia local is effective and sure. ( Rev Med Hered 2000; 11: 03-06 ).

KEY WORD: Haemorrhoidectomy, hemorrhoidal pathology.

\section{RESUMEN}

Objetivo: Evaluar la utilidad de la Cirugía ambulatoria con anestesia local en pacientes con patología hemorroidal. Material y métodos: Se realizó un estudio prospectivo, transversal, entre Abril de 1996 y Marzo de 1999, en el servicio de Cirugía de la Clínica Fiori. Se intervinieron 260 pacientes por patología hemorroidal, de ellos 229 pacientes fueron sometidos a hemorroidectomía con anestesia local, los que fueron incluidos en el estudio. La técnica quirúrgica utilizada fue la de Milligan y Morgan con un tiempo operatorio de 20 minutos; y control a los 3 días. Resultados: El 69.4\% de patología hemorroidal se observó entre los 31-60 años de edad. Sólo el 1.7\% (4 pacientes) presentaron complicaciones post-operatorias, 2 pacientes con sangrado leve y 2 con hemorroides residuales, ninguno resultó ser de gravedad; y el $98.3 \%$ (225 pacientes) no presentaron ningún tipo de complicaciones. No hubo accidentes anestésicos. Conclusión: La hemorroidectomía con anestesia local es efectiva y segura. ( Rev Med Hered 2000; 11: 03-06 ).

PALABRAS CLAVE: Hemorroidectomía, hemorroides.

* Médico Cirujano General-ESSALUD. Profesor Auxiliar de Anatomía Humana. Departamento de Ciencias Morfológicas-Universidad Peruana Cayetano Heredia.

** Profesor Auxiliar, Escuela de Tecnología Médica. Universidad Peruana Cayetano Heredia. 


\section{INTRODUCCION}

La patología hemorroidal es una de las mas frecuentes dentro de las enfermedades ano-rectales conocidas (52$60 \%$ ), aunque su incidencia no es precisa y es variable según otros autores (1) lo que implica que el tratamiento en su mayoría quirúrgico debe ser rápido, eficaz y a bajo costo, siendo esto el reto del presente trabajo.

En 1909 surge el concepto de Cirugía de día establecido por Nicoll, en el Hospital Royal Glasgow; donde se reportan a partir de esa fecha procedimientos quirúrgicos en pacientes ambulatorios que evolucionaron satisfactoriamente. $(2,3,4,5,6)$.

En países desarrollados de Europa, Estados Unidos y Canadá la cirugía ambulatoria crece a un ritmo exponencial, se espera que el $60 \%$ de toda la cirugía será efectuada ambulatoriamente (7). Creemos que este porcentaje podría ser mayor con mejoras en las técnicas quirúrgicas, evolución en anestesiología, avances en monitoreo de pacientes y un buen laboratorio. La crisis económica de nuestro país deja fuera de cuestionamiento la utilidad de la cirugía ambulatoria en la solución de los problemas quirúrgicos, permitiendo el desarrollo de nuevas técnicas que faciliten la ejecución de procedimientos que, hasta ahora, requerían la permanencia de los pacientes por lo menos durante una noche en el hospital luego de la intervención quirúrgica.

En este trabajo se analiza la experiencia efectuada en la especialidad de Cirugía General de la Clínica Fiori, de la hemorroidectomía con anestesia local, siendo el objetivo principal evaluar la utilidad de este tipo de anestesia en los pacientes con patología hemorroidal, para la mejora de atención en pacientes sometidos a Cirugía ambulatoria en la Clínica Fiori.

\section{MATERIAL Y METODOS}

Dentro del presente estudio de investigación prospectivo, transversal y descriptivo, se incluyeron a pacientes comprendidos desde Abril de 1996 hasta Marzo de 1 999. Se intervinieron 260 pacientes sometidos a hemorroidectomía, de los cuales 229 pacientes se intervinieron con anestesia local más sedación y 31 pacientes con anestesia general, los que se excluyeron del estudio. Los 229 pacientes presentaron hemorroides externas, internas y mixtas.

En el pre oporatorio se tomó en cuenta: a) análisis de laboratorio: hemograma completo, hemoglobina, tiempo de coagulación y sangría, VDRL, glucosa, creatinina, examen completo de orina y en pacientes mayores de 40 años se agregó riesgo quirúrgico; b) preparación psicológica y educación al paciente sobre la técnica quirúrgica a utilizarse y tipo de anestesia; c) indicaciones precisas al paciente sobre su preparación física desde el día anterior a la operación; d) examen clínico general exhaustivo; e) consentimiento escrito por los pacientes.

En el operatorio se tuvo las siguientes características:

a) sin premedicación anestésica; b) lavado de zona operatoria con agua y jabón y luego aplicación con yodopovidona; c) anestésicos de uso del anestesiólogo: Penthotal, Fentanyl, Diazepam, ketamina, Halotano; d) anestésicos de uso del cirujano: lidocaina $2 \% \mathrm{~s} / \mathrm{Ep}$. diluído con $20 \mathrm{cc}$ de ClNa 0.9\%.

La técnica anestésica que se empleó fue la infiltración local alrededor del margen anal e infiltración del esfínter anal externo e interno y la técnica operatoria utilizada fue la técnica abierta o técnica de Milligan y Morgan; con un tiempo operatorio de 20 minutos.

Todos los pacientes fueron dados de alta a las 2 horas de la intervención, recibiendo indicaciones médicas precisas tanto para el paciente como el familiar acompañante. Los pacientes fueron controlados a los 3 días por consulta externa.

\section{RESULTADOS}

En la tabla $\mathrm{N}^{\circ} 1$ se puede apreciar que el grupo etáreo comprendido entre los 31 años y 60 años (69.4\%) constituyen el mayor número de pacientes con hemorroides.

En la tabla $\mathrm{N}^{\circ} 2$, se muestra el tipo de complicaciones postoperatorias tanto mediatas como inmediatas, con su respectivo tratamiento, ninguno de ellos resultó ser de gravedad.

Sólo 4 pacientes $(1.7 \%)$ presentaron complicaciones luego de la intervención quirúrgica, mientras que 225 pacientes $(98.3 \%)$ no tuvieron ningún tipo de complicaciones (Figura $\mathrm{N}^{\circ} 1$ ). No hubo ningún accidente anestésico, ni de otro tipo durante las intervenciones.

\section{DISCUSION}

Las hemorroides afectan casi al $4 \%$ de la población en general (8), aumentando su frecuencia al parecer con la edad y parece probable que por lo menos el $50 \%$ de las personas mayores de 50 años sufran cierto grado de desarrollo hemorroidal (9), lo cual se puede observar también en nuestro estudio. 


\begin{tabular}{lcc}
\hline \multicolumn{3}{l}{ Tabla $\mathbf{N}^{\circ}$ 1. Pacientes según edad. } \\
\hline Edad & Pacientes & $\%$ \\
\hline $20-30$ & 36 & 15.72 \\
$31-40$ & 58 & 25.33 \\
$41-50$ & 55 & 24.02 \\
$51-60$ & 46 & 20.09 \\
61 ó más & 34 & 14.84 \\
& & \\
TOTAL & 229 & 100.00 \\
\hline
\end{tabular}

Las hemorroides son dilataciones circunscritas del vaso venoso de la región perianal y canal anal (10).

Estas pueden ser externas: por debajo de la línea pectínea, cubiertas por piel que se diagnostican por la inspección; e internas: situadas por encima de la línea pectinea, cubiertas por mucosa, que se diagnostican por anoscopía, salvo en casos de prolapso o trombosis (9).

Existen varias formas de tratamiento, siendo la hemorroidectomia quirúrgica la mas utilizada, no por ser la mejor, sino por el grado avanzado de desarrollo hemorroidal que presentan los pacientes durante la consulta. Aunque existen estudios a favor de la técnica cerrada o de Ferguson con anestesia local con un bajo porcentaje de complicaciones (11), en nuestro estudio se realiza la técnica abierta o de Milligan y Morgan con anestesia local que muestra solo $1.5 \%$ de complicaciones.

Dentro de las complicaciones más frecuentes de la hemorroidectomía quirúrgica, se describen la retención urinaria, impactación fecal que se produce varios días después de la operación $(1,12)$, la cual no fue observada en nuestro estudio. Además, se describe sangrado postquirúrgico con una frecuencia del $2 \%(1,12)$; en nuestro estudio encontramos $0.9 \%$ de complicaciones por sangrado. También se menciona como complicación la estenosis en menos de $1 \%$ de los casos (1), que no fue observado en nuestro estudio. Lo mejor es evitar la retención urinaria mediante restricción de líquidos durante el procedimiento quirúrgico. También experimentan problemas con la micción los que se han sedado en grado excesivo (1).

\section{Tabla N². Complicaciones post-operatorias.}

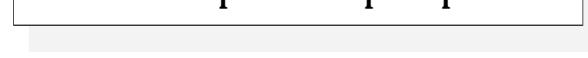

\begin{tabular}{lcc} 
Complicación & Pacientes & Tratamiento \\
\hline $\begin{array}{l}\text { Sangrado leve } \\
\text { post hemorroidectomía }\end{array}$ & 2 & Revisión hemostática \\
Hemorroides residuales & 2 & Reintervención \\
TOTAL & & quirúrgica \\
\hline
\end{tabular}

La experiencia venezolana, señala la utilidad del procedimiento ambulatorio en cirugía general y, también en forma muy tímida, en la cirugía anal $(13,14,15)$.

Pero se ha observado que la mayor parte de los procedimientos quirúrgicos por lesiones del conducto anal se adapta fácilmente al ejercicio del consultorio, entre ellos la hemorroidectomía (1). Conforme el médico adapta mejor sus servicios para satisfacer las necesidades de la sociedad, los pacientes van aceptando en mayor grado el alta temprana. Teóricamente cualquier paciente es bueno para irse a casa después de la intervención, si ésta ha transcurrido sin ningún tropiezo. Por consiguiente, un requisito indispensable es que el paciente, sobre todo si es de alto riesgo, esté en condiciones estables para el momento de la cirugía.

\section{Figura N¹. Complicaciones post-hemorroidectomía.}

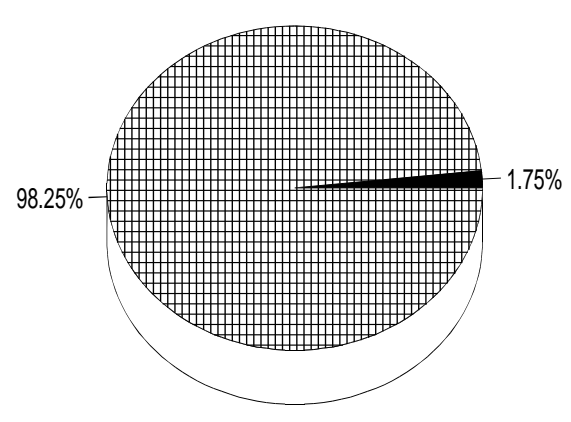

Con complicación $\mathbb{\boxplus}$ Sin complicación 
Si estas condiciones son iguales después de la intervención, no hay razón alguna para que el paciente deba quedarse hospitalizado $(1,13)$.

Cualquier anestesia es adecuada para la cirugía ambulatoria, exceptuando la anestesia raquídea por ser la más proclive a complicaciones, particularmente cefaleas. El anestesiólogo, de acuerdo con el paciente, escogerá la más apropiada (13). Gracias al uso de la anestesia local de la misma manera que se aplicaría en la sala de operaciones, es posible realizar la mayor parte de los procedimientos, en el consultorio o en un centro de cirugía ambulatoria (1).

Es importante que todos los pacientes se envíen al hogar con instrucciones verbales y escritas apropiadas, y que éstas se conviertan en parte del expediente médico para la protección del paciente, lo mismo que del cirujano. Los pacientes pueden ser llamados por su cirujano 24 horas después del alta (1), ó tener el primer control post-operatorio dentro de las 48 horas después de la intervención. (13). En nuestro estudio se realizó a los 3 días de la intervención.

Se concluye como en otros reportes $(11,12,16)$, que la hemorroidectomía con anestesia local, resulta ser confortable y bien tolerada por los pacientes, brindando un reintegro temprano al trabajo, además, no representa ningún riesgo para los pacientes.

\section{Correspondencia:}

Dr. Noel Aliaga.

Dpto. de Ciencias Morfólogicas, Facultad de Medicina Alberto Hurtado.

Universidad Peruana Cayetano Heredia. Av. Honorio Delgado 430 San Martin de Porres. Lima - Perú

\section{BIBLIOGRAFIA}

1. Mazier WP. Hemorroides, fisura y prurito anal Clin Quir Norteamérica 1996; 4:1339-1352

2. Detmer D et aL Cirugía en pacientes ambulatorios. Clin Quir Norteamérica 1982; 4: 691-711.

3. Detmer D. Ambulatory surgery. N Engl J Med 1981; 305: 1406-1409.

4. Ogg T. Aspects of day surgery and anaesthesia. Anaesth Rounds 1985; 18: 3-27

5. Reed W Ford J. El Centro Quirúrgico, Clin Obstet Gynecol NA 1974; Set 215-227.

6. Oosterlee J. Surgery in out patients. Sr Med J 1979; Dec.- 1459-1460.

7. Bemal 0. Unidad ambulatoria:Estado actual. Act Per Anest 1991; 6: 61-71

8. Argov S. Radical ambulatory hemorhioidectomy. Harefuah 1994; 126:189-191.

9. Ben Vitt DP. Varicose veins, deep vein thrombosis and hemorroids. Epidemiology and suggested etiology. $\mathrm{Br}$ Med J 1972; 2:556

10.Robbins. Tratado de Patologia Ed Interamericana 1963: 694.

11.Ochoa F, Bozza V, Alfaro R, Sigala H. Hemorroidectomía cerrada con anestesia local vs hemorroidectomía abierta: Estudio prospectivo y comparativo. Rev Venez Cir 1993; 46: $94-100$

12.Lacerda A, Cunha JR. Outpatient haemorrhoidectomy under local anaesthesia. Eur J Surg 1997; 163:935-40.

13. Morgado PJ, Manrique 0. Cirugía ambulatoria en coloproctología. Gac Med Caracas 1995; 103: 247- 250.

14.Kiríakidis C, Kiriakidís JL, Berlanga L, Berlanga MK. Cirugía ambulatoria: experiencia de 6 años. Rev Venez Cir 1994; 47:59-64.

15.Romero RA, Tremarias DA, Peroza SE, Caraballo AR. Cirugía ambulatoria. Experiencia en el Hospital Distrital “Dr. Miguel Malpíca Jiménez”. Rev Venez Cir 1994; 47:6975.

16.Celoria G, Falco E, Nardini A, Gianardi M, Poletti G, Di Alesio L. Local anaesthesia in anal surgery. Technical note and inmediata results. Minerva Chir 1993; 48: 11031106. 